\title{
An Insight into the Interactions between $\alpha$-Tocopherol and Chitosan in Ultrasound-Prepared Nanoparticles
}

\author{
Majid Naghibzadeh, ${ }^{1}$ Amir Amani, ${ }^{1}$ Mohsen Amini, ${ }^{2}$ Elina Esmaeilzadeh, ${ }^{1}$ \\ Negar Mottaghi-Dastjerdi, ${ }^{2}$ and Mohammad Ali Faramarzi ${ }^{2,3}$ \\ ${ }^{1}$ Department of Medical Nanotechnology, Faculty of Advanced Medical Technologies, Tehran University of Medical Sciences, \\ Tehran 14174, Iran \\ ${ }^{2}$ Department of Pharmaceutical Biotechnology, Biotechnology Research Center, Faculty of Pharmacy, \\ Tehran University of Medical Sciences, P.O. Box 14155-6451, Tehran 14174, Iran \\ ${ }^{3}$ Biotechnology Research Center, Tehran University of Medical Sciences, Tehran 14174, Iran
}

Correspondence should be addressed to Mohammad Ali Faramarzi, faramarz@tums.ac.ir

Received 31 January 2010; Accepted 6 June 2010

Academic Editor: Doron Yadlovker

Copyright (C) 2010 Majid Naghibzadeh et al. This is an open access article distributed under the Creative Commons Attribution License, which permits unrestricted use, distribution, and reproduction in any medium, provided the original work is properly cited.

\begin{abstract}
The aim of this study was to investigate the interactions between $\alpha$-tocopherol and chitosan molecules prepared subsequent to preparation of $\alpha$-tocopherol-loaded chitosan nanoparticles using ultrasonication. Dynamic light scattering (DLS) and scanning electron microscopy (SEM) analyses showed semispherical particles with an average size of approximately $350 \mathrm{~nm}$. Also from reconstitution test, $\alpha$-tocopherol was suggested as stabilizing agent during lyophilization/reconstitution process. The zeta potentials of chitosan and $\alpha$-tocopherol nanoparticles were larger than $\pm 30 \mathrm{mV}$, representing suitable stability. Data obtained from FTIR showed possibility of chemical interaction between chitosan and $\alpha$-tocopherol. Furthermore, the results from FTIR, NMR, and XRD spectroscopy confirmed electrostatic interactions between the two molecules. Overall, this procedure could be considered as a facile method to prepare $\alpha$-tocopherol-loaded nanoparticles.
\end{abstract}

\section{Introduction}

Chitosan, the second plentiful polysaccharied after cellulose in nature, is a biopolymer derived by alkaline deacetylation of chitin [1]. This polymer, exhibiting unique properties such as biocompatibility, biodegradability, low cytotoxicity, bioadhesivity, and the ability to increase the drugs absorption through polyanionic membranes, has attracted attention of many researchers in drug delivery systems $[2,3]$. Among different types of chitosans, medium and high molecular weight chitosans are not fully dispersible in neutral aqueous media. This in turn limits the application of chitosans in delivery of $\mathrm{pH}$-sensitive active agents such as proteins, enzymes, and genes [4]. During recent years, among different types of chitosans, low molecular weight chitosan has gained attention as a drug conjugate according to its water solubility without the need for low $\mathrm{pH}$ values $[5,6]$.
$\alpha$-Tocopherol, one of the lipid soluble vitamins, has proved useful in anticancer, anti-inflammatory, and antioxidant activities [7]. However, conventional formulations of tocopherol often fail to provide satisfactory bioavailability due to its hydrophobic characteristics [8]. To overcome this problem, based on Bruner and Tolloczko equation [9], it could be hypothesized that increasing the particles surface area leads to an increase in the dissolution velocity [9]. This hypothesis had been confirmed by Kotyla and coworkers that had compared nanosized tocopherol with microsized particles of this drug. The results showed an increase in bioavailability of tocopherol [10].

The principal of ultrasonication is based on formation, growth, and implosion of acoustic cavity. Ultrasonication is a green, simple, fast, popular, and reproducible method. In this study, we used low frequency ultrasonication for preparation of $\alpha$-tocopherol-loaded chitosan nanoparticles. The study 
aimed to investigate the possibility of loading $\alpha$-tocopherol on the chitosan nanoparticles followed by determining interactions between the drug and the nanocarrier.

\section{Experiments}

2.1. Chemicals. Water soluble chitosan $(\mathrm{MW}<18 \mathrm{KD}$, degree of deacetylation $=86 \%$ ) was gifted from Easter Holding Group (China). $\alpha$-Tocopherol, deuterium oxide $\left(\mathrm{D}_{2} \mathrm{O}\right)$, and dimethyl sulfoxide (DMSO) were of analytical grades and purchased from Merck (Darmstadt, Germany).

2.2. Preparation of $\alpha$-Tocopherol-Loaded Chitosan Nanoparticles. Fifty $\mathrm{mg}$ of low molecular weight chitosan was dispersed in $10 \mathrm{~mL}$ of deionized water. A specified amount of $\alpha$ tocopherol was dispersed in $1 \mathrm{~mL}$ of ethanol and dropped into chitosan solution. The mixtures were placed in ice bath and sonicated using the probe ultrasonication (Lab Sonic, B. Braun, Germany) at $20 \mathrm{KHz}$ to form $\alpha$-tocopherolloaded chitosan nanoparticles. Subsequently, ethanol was evaporated using rotary evaporator, and the prepared samples were lyophilized for future analyses. All procedures were performed in darkened condition. Four weight ratio (mg/mg) of chitosan to $\alpha$-tocopherol, namely, 50/2 (i.e., Ch50/2), 50/5 (i.e., Ch50/5), 50/10 (i.e., Ch50/10), and 50/20 (i.e., Ch50/20) were prepared using ultrasonication.

2.3. Particle Size and Zeta Potential Measurement. Dynamic light scattering (DLS) studies were performed using Zetasizer 3000 (Malvern Instruments, UK) at $25^{\circ} \mathrm{C}$ to obtain the particle size and zeta potential of the particles. Each measurement was repeated three times. No dilution was made to the samples, and the viscosity of water was taken as the viscosity of dispersant.

2.4. Scanning Electron Microscopy. To study the morphology of $\alpha$-tocopherol-loaded nanoparticles, scanning electron microscopy (SEM) was employed. The suspension was gold coated and placed on a sputter and a DSM, $960 \mathrm{~A}$; Zeiss (Germany) operating at $10 \mathrm{kV}$ was used to visualize the nanoparticles.

2.5. Reconstitution Test. To investigate the possible changes in the particles properties before lyophilization and after reconstitution, for each chitosan $/ \alpha$-tocopherol weight ratio (i.e., Ch50/2, Ch50/5, Ch50/10, and Ch50/20), $10 \mathrm{mg}$ of final lyophilized powder was dissolved in $10 \mathrm{~mL}$ of deionized water and vortexed for $10 \mathrm{~s}$. The zeta potential and particle size of the samples were then measured using the method described in Section 2.3.

\subsection{Fourier-Transform-Infrared Spectroscopy. Chemical} interactions and structure of chitosan and $\alpha$-tocopherol in nanoparticles were studied using Fourier-transform-infrared (FT-IR) spectroscopy employing $\mathrm{KBr}$ discs on an FT-IR spectrometer (Shimadzu, FT-IR 8700, Japan).

2.7. ${ }^{1} H$ Nuclear Magnetic Resonance Spectra Studies. The interactions between chitosan and $\alpha$-tocopherol were fur- ther investigated using ${ }^{1} \mathrm{H}$ nuclear magnetic resonance $\left({ }^{1} \mathrm{H}\right.$ NMR) spectroscopy. The chitosan and $\alpha$-tocopherol nanoparticles were dissolved in dimethyl sulfoxide (DMSO) and deuterium oxide $\left(\mathrm{D}_{2} \mathrm{O}\right) .{ }^{1} \mathrm{H}$ NMR spectra were recorded on a Bruker DRX 400 spectrometer, (500 MHz, USA) operating at a probe temperature of $298^{\circ} \mathrm{K}$.

2.8. X-Ray Diffraction. The powder X-ray diffraction (XRD) was performed by an X'Pert MPD Powder X-ray Diffraction System (Philips, Holland) using $\mathrm{Cu} \mathrm{K} \alpha$ radiation $(40 \mathrm{kV}$, $40 \mathrm{~mA}$ ) to study chemical structure of nanoparticles.

\section{Results and Discussion}

3.1. Characterization of $\alpha$-Tocopherol and Chitosan Nanoparticles. $\alpha$-Tocopherol is a hydrophobic compound which is practically insoluble in water $(<0.1 \mathrm{mg} / \mathrm{mL})$ [11]. Thus, conventional formulation of this active agent will lead to low bioavailability when taken orally. Water soluble chitosan, with high contents of free-amine groups, has been reported suitable for solubilization of hydrophobic compounds like all-transretinoic acid through establishing ionic complexes between the two molecules [5]. Dissolution velocity as the second factor that affects bioavailability is also affected by the particle size. According to Noyes and Whitney as well as Bruner and Tolloczko equations, reducing particle size directly affects the dissolution rate and bioavailability of the active agent [10]. Therefore, a proper nanoparticles formulation of chitosan that is able to solubilize $\alpha$-tocopherol will be able to increase its bioavailability.

In this paper, we used low molecular weight chitosan which takes the advantage of being water soluble. In contrast to other chitosans which require acidic conditions to be dissolved, low molecular weight chitosan can be easily dissolved in neutral aqueous media and thus be used for delivery of wider range of drugs including $\mathrm{pH}$-sensitive ones.

The different formulations were subjected to DLS particle size analysis. The particle size and zeta potential values obtained for $\alpha$-tocopherol-loaded nanoparticles 30 minutes after preparation are shown in Table 1. The data shows that by increasing the $\alpha$-tocopherol content in the formulation, a sharp increase in the particle size can be observed when increasing the $\alpha$-tocopherol content from 2 to 5 , followed by a slight decrease in the observed particle size in further increases of the $\alpha$-tocopherol content. One explanation for this observation is that when in low value of $\alpha$-tocopherol amount in the formulation (i.e., Ch50/5), the excess $\alpha$ tocopherol molecules will mainly deposit on the surface of the chitosan particles leading to an increase in the observed hydrodynamic sizes. However, by further increase in the $\alpha$-tocopherol content, the $\alpha$-tocopherol molecules migrate to the internal parts of the nanoparticles, establishing proper interactions between the two molecules which lead to molecules rearrangements in the molecular structure of nanoparticles to make more stable particles. This rearrangement will be the main cause for the observed decrease in the particle size. This hypothesis appears to be applicable due to the fact that ultrasonication can render the rearrangement in the molecular structure of particles [12] and has been 


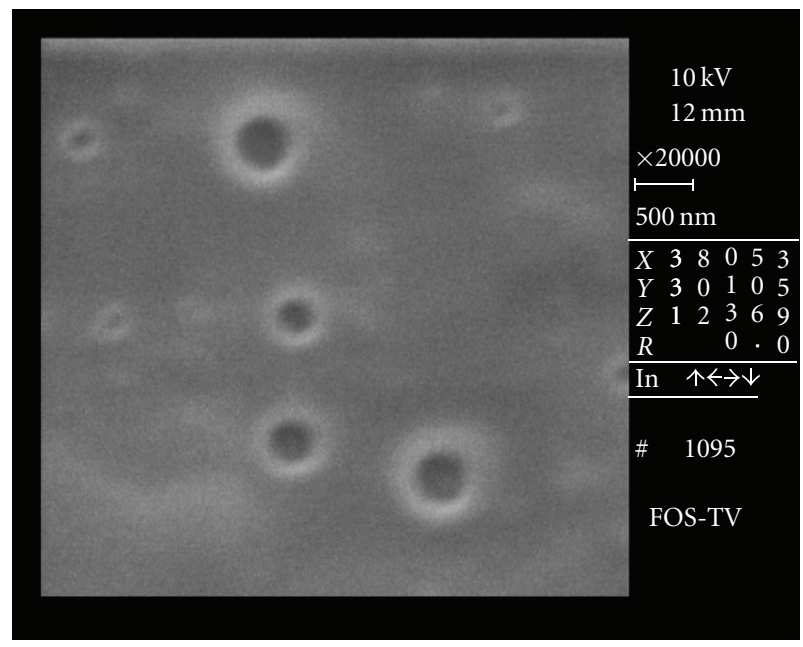

FIGURE 1: SEM images of chitosan and $\alpha$-tocopherol nanoparticles.

TABLE 1: Characterization of freshly prepared $\alpha$-tocopherol and chitosan nanoparticles.

\begin{tabular}{lcccc}
\hline Chitosan/ $\alpha$-tocopherol & $\begin{array}{c}\text { Weight ratio } \\
(\mathrm{mg} / \mathrm{mg})\end{array}$ & $\begin{array}{c}\text { Average } \\
\text { particle size } \\
(\mathrm{nm}, n=3)\end{array}$ & Zeta potential $(\mathrm{mV})$ & Polydispersity Index \\
\hline Ch50/2 & $50 / 2$ & 277 & $-76.8 \pm 4.8$ & 0.276 \\
Ch50/5 & $50 / 5$ & 378 & $-74.0 \pm 5.4$ & 0.197 \\
Ch50/10 & $50 / 10$ & 358 & $-61.7 \pm 8.7$ & 0.253 \\
Ch50/20 & $50 / 20$ & 348 & $-61.5 \pm 4.1$ & 0.245 \\
\hline
\end{tabular}

verified by the zeta potential and reconstitution tests as will be discussed later. The polydispersity index is another factor that represents the dispersion homogeneity. The prepared nanoparticles exhibited relatively narrow particle size distribution, as indicated by a relatively low polydispersity index (PDI) values with all values being less than 0.3 (Table 1 ).

Tables 1 and 2 indicate zeta potential changes before and after reconstitution of the four samples. In our study, the zeta potentials of all studied samples were larger than $\pm 30 \mathrm{mV}$ (see Table 1), and thus acceptable stability can be predicted for the samples. Also, as can be observed from Table 1, the zeta potential has decreased by increasing the $\alpha$-tocopherol content in the formulation. As $\alpha$-tocopherol is probably the major cause for the change in the zeta potential of the nanoparticles, it could be suggested that the considerable "jump" observed in the zeta potential of Ch50/5 compared to Ch50/10 indicates the insertion of the $\alpha$-tocopherol molecules into the chitosan particles, causing the zeta potential to decrease from -74.0 to $-61.7 \mathrm{mV}$. Also, as shown in Table 2, zeta potential and particle size of reconstituted $\alpha$-tocopherol-loaded nanoparticles were similar to original preparation when having more than $10 \mathrm{mg}$ of $\alpha$-tocopherol in the formulation which also suggests that $\alpha$-tocopherol plays an important role in stabilizing the chitosan nanoparticles.

Morphology of the chitosan and $\alpha$-tocopherol nanoparticles was characterized by scanning electron microscope
(SEM) (see Figure 1). The SEM images indicated that nanoparticles were nearly monodisperse with semispherical shapes. The observed particles size are also almost similar to the particle size results from DLS.

\subsection{Reconstitution of $\alpha$-Tocopherol and Chitosan Nanopar-} ticles. One of the factors that can potentially affect the nanoparticles properties is the freeze-drying procedure. It has been reported that additives such as saccharides are usually essential for cryoprotection of the nanoparticles in the lyophilization procedure [13]. Based on our results in reconstitution test, when lyophilized nanoparticles were reconstituted into water (Table 2 ), the particle size and distribution of the particle size were practically similar to original aqueous solution (Table 1 ) when the $\alpha$-tocopherol to chitosan ratio was more than $10 / 50$ (i.e., Ch50/20 and Ch50/10). This observation can confirm the hypothesis of $\alpha$-tocopherol molecules inserting the chitosan nanoparticles and stabilizing them. As a result, it could be argued that the $\alpha$-tocopherol-loaded chitosan nanoparticles can be successively lyophilized and reconstituted without the need to a cryoprotectant.

Stability of solution is an essential factor in marketing. Klaypradit and Huang indicated that chitosan alone is unable to produce stable emulsions, showing the need for a surfactant when designing chitosan preparations [14]; 
TABLE 2: Characterization of $\alpha$-tocopherol and chitosan nanoparticles after reconstitution.

\begin{tabular}{lcccc}
\hline Chitosan/ $\alpha$-tocopherol & $\begin{array}{c}\text { Weight ratio } \\
(\mathrm{mg} / \mathrm{mg})\end{array}$ & $\begin{array}{c}\text { Average } \\
\text { particle size } \\
(\mathrm{nm}, n=3)\end{array}$ & Zeta potential $(\mathrm{mV})$ & Polydispersity \\
\hline Ch50/2 & $50 / 2$ & 383 & $-48.7 \pm 7.6$ & 0.360 \\
Ch50/5 & $50 / 5$ & 325 & $-57.3 \pm 4.9$ & 0.192 \\
Ch50/10 & $50 / 10$ & 330 & $-61.5 \pm 9.6$ & 0.258 \\
Ch50/20 & $50 / 20$ & 351 & $-61.2 \pm 5.3$ & 0.237 \\
\hline
\end{tabular}

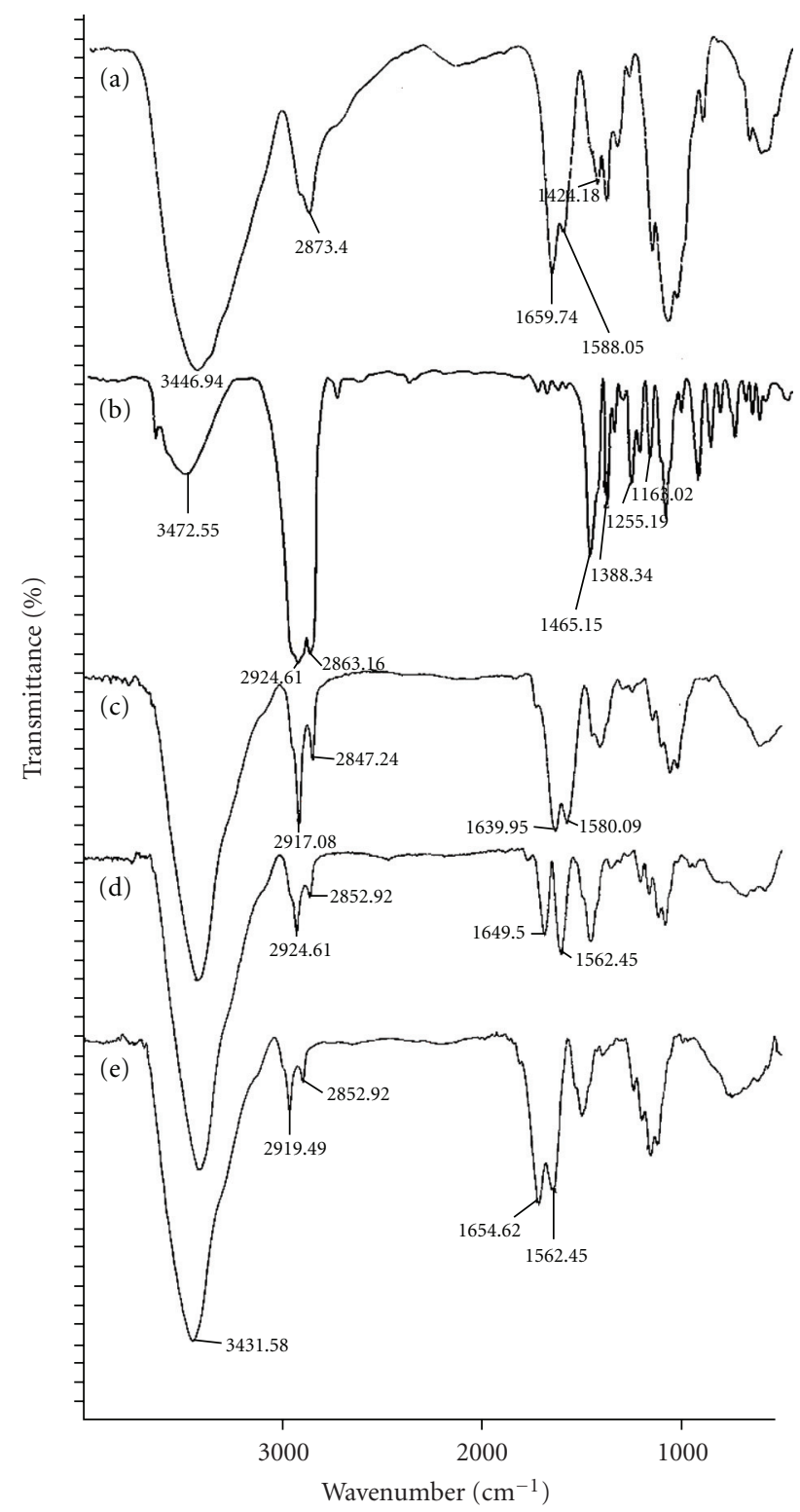

Figure 2: FTIR spectra of (a) chitosan, (b) $\alpha$-tocopherol, (c) Ch50/2, (d) Ch50/5, and (e) Ch50/20.

however based on our results in reconstitution test, $\alpha$ tocopherol-loaded chitosan nanoparticles were completely reconstituted into neutral aqueous solution without adding cryoprotectants, indicating economical benefits in scale up.
3.3. Analysis of $\alpha$-Tocopherol and Chitosan Nanoparticles. Figure 2 shows the FTIR spectra obtained for $\alpha$-tocopherol, chitosan and $\alpha$-tocopherol-loaded chitosan. In Figure 2(a) the broadband at around $3446 \mathrm{~cm}^{-1}$ attributes to $-\mathrm{NH}$ and $-\mathrm{OH}$ stretching vibration. The weak band at $2873 \mathrm{~cm}^{-1}$ may 


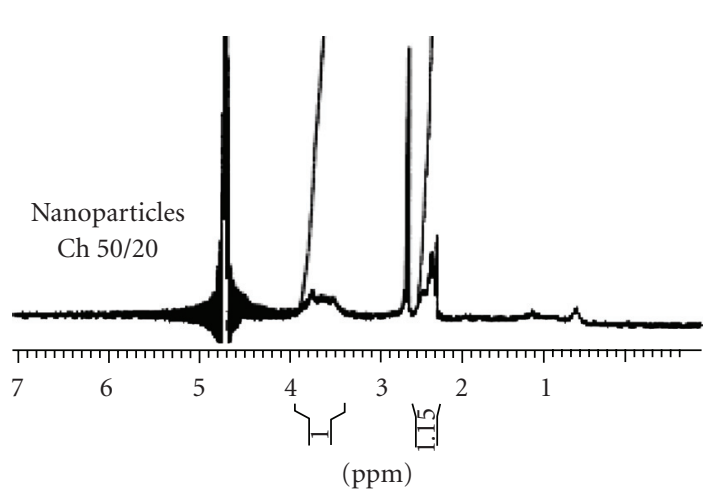

(a)

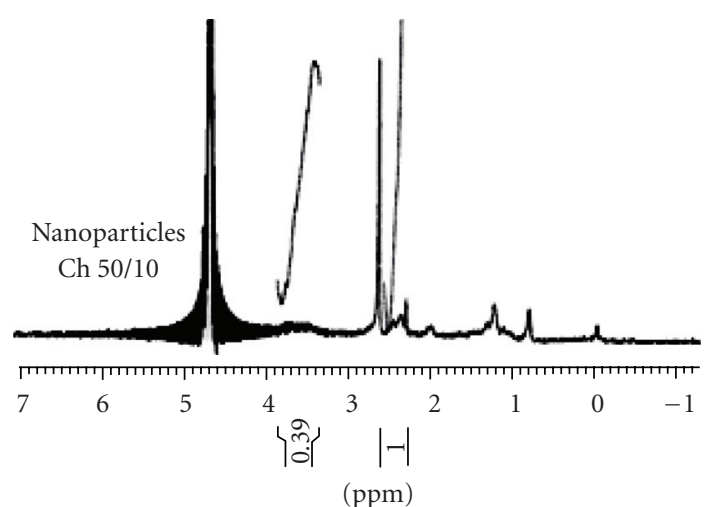

(b)

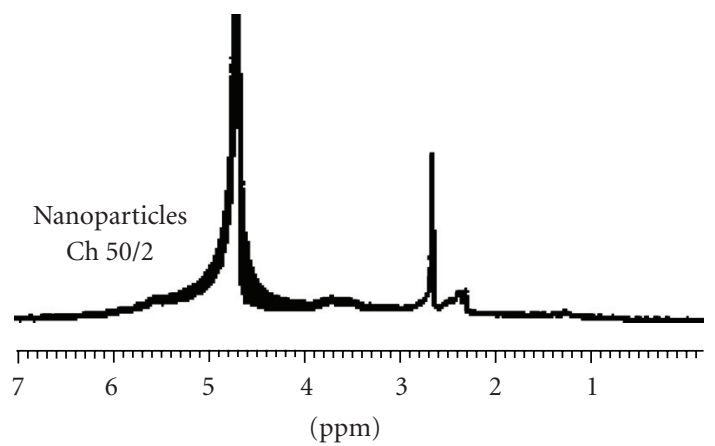

(c)

FIgURE 3: ${ }^{1} \mathrm{H}$ NMR spectra of $\alpha$-tocopherol-loaded chitosan nanoparticles.

be due to $-\mathrm{CH}-$ stretching in chitosan. The bands at 1659 and $1590 \mathrm{~cm}^{-1}$ represent amide and amine groups of chitosan, respectively, and the bands at $1070 \mathrm{~cm}^{-1}$ are assigned to the skeletal vibration of $\mathrm{C}-\mathrm{O}$ stretching [15].

Figure 2(b) shows the spectrum of $\alpha$-tocopherol. The band at $3472 \mathrm{~cm}^{-1}$ is associated with $-\mathrm{OH}$ as has been reported previously in [16]. Also, bands at 2924 and $2863 \mathrm{~cm}^{-1}$ represent the asymmetric and symmetric stretching vibrations of the $-\mathrm{CH}_{2}-$ and $-\mathrm{CH}_{3}$, respectively, $1465 \mathrm{~cm}^{-1}$ is for phenyl, skeletal and methyl asymmetric bending, $1388 \mathrm{~cm}^{-1}$ is associated with methyl symmetric bending, $1081 \mathrm{~cm}^{-1}$ is attributed with plane bending of phenyl and $922 \mathrm{~cm}^{-1}$ stands for trans $=\mathrm{CH}_{2}$ stretching.

Comparing Figures $2(\mathrm{a})$ and $2(\mathrm{c})-2(\mathrm{e})$, the peak at $1590 \mathrm{~cm}^{-1}$ for primary amine bending has been shifted to new positions, $1580 \mathrm{~cm}^{-1}$ and 1562 , most probably due to the electrostatic interactions occurring for $-\mathrm{NH}_{2}$ group of chitosan with $\alpha$-tocopherol. On the other side, the peak of $3472 \mathrm{~cm}^{-1}$ from $\alpha$-tocopherol (i.e., Figure 2(b)) has been disappeared in Figures 2(c)-2(e). This may be due to $-\mathrm{OH}$ being engaged in an interaction with chitosan, thus shifting the associated peak value to lower values and being located behind the large peak of $3431 \mathrm{~cm}^{-1}$ observed in Figures 2(c)2 (e). In general, from FTIR results, it can be suggested that a chemical interaction between $-\mathrm{NH}_{2}$ group of chitosan and $\mathrm{OH}$ group of $\alpha$-tocopherol has been occurred. Considering the molecular structure of $\alpha$-tocopherol and chitosan, an electrostatic interaction bond between $-\mathrm{OH}$ and $-\mathrm{NH}_{2}$ seems reasonable here.

To further investigate the properties of formed nanoparticles, ${ }^{1} \mathrm{H}$ NMR spectroscopy was employed (see Figure 3 ). In the ${ }^{1} \mathrm{H}$ NMR spectra, the broad multiplet peaks from 2.2 to $2.6 \mathrm{ppm}$ are attributed to $\alpha$-tocopherol [17]. Also peaks from 3.3 to $3.9 \mathrm{ppm}$ correspond to protons of glucose amine in chitosan [15].

The X-ray diffraction studies were also performed to compare the structure of $\alpha$-tocopherol and chitosan nanoparticles with the mixture of $\alpha$-tocopherol and chitosan as shown in Figure 4. Chitosan spectrum showed six peaks at approximately $20^{\circ}, 28^{\circ}, 29^{\circ}, 30^{\circ}, 34^{\circ}, 36^{\circ}$, and $54^{\circ}$, with strongest reflection at $2 \theta \approx 20^{\circ}$ (see Figure 4(b)). $\alpha$ Tocopherol spectrum indicated strongest reflection at $2 \theta \approx$ $19^{\circ}$ (Figure $4(\mathrm{a})$ ). The physical mixture of $\alpha$-tocopherol and chitosan spectrum, having chitosan $/ \alpha$-tocopherol weight ratio of $1: 1$ (Figure $4(\mathrm{c})$ ) indicates strongest reflection at $2 \theta \approx 19.5^{\circ}$ and specific peaks at $2 \theta \approx 28^{\circ}, 36^{\circ}$ and $46^{\circ}$. The XRD results for $\alpha$-tocopherol-loaded nanoparticles show that by increasing the $\alpha$-tocopherol content, the peak at $2 \theta \approx 22^{\circ}$ shifts towards the lower values, becoming closer to the peak of unconjugated $\alpha$-tocopherol (i.e., $2 \theta \approx$ $19^{\circ}$ as observed in Figure 4(a)). Also specific sharp crystal peaks, observed in physical mixture, can not be observed 
(a)

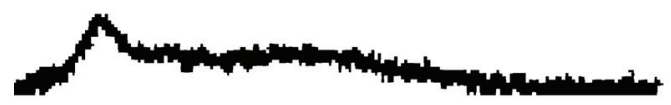

(b)

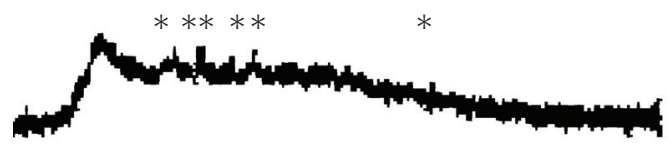

(c)

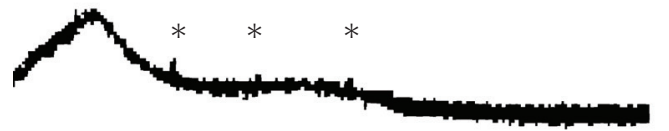

(d)

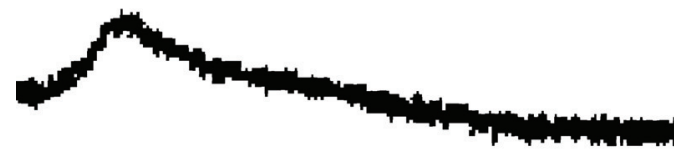

(e)

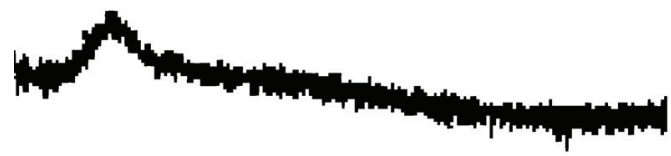

(f)

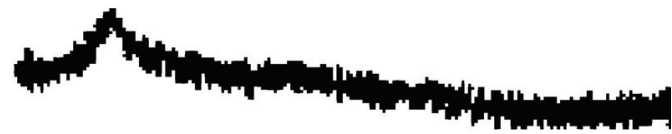

(g)

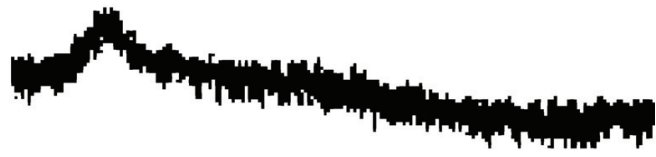

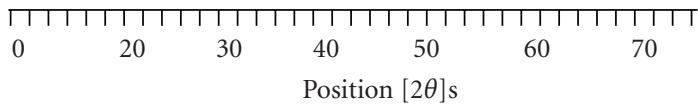

Figure 4: X-ray diffraction pattern of (a) $\alpha$-tocopherol, (b) chitosan, (c) physical mixture of $\alpha$-tocopherol and chitosan, (d) Ch50/2, (e) Ch50/5, (f) Ch50/10, and (g) Ch50/20.

at nanoparticles spectrum, indicating structural differences. From the XRD results, it can be concluded that not only $\alpha$ tocopherol molecules were present in chitosan nanoparticles; but also by introducing $\alpha$-tocopherol molecules to chitosan particles, a change in the crystal structure has been occurred, indicating chemical interactions between $\alpha$-tocopherol and chitosan molecules.

\section{Conclusion}

This study showed the ability of $\alpha$-tocopherol molecules to interact with and stabilize the chitosan nanoparticles. $\alpha$ tocopherol-loaded nanoparticles were prepared by ultrasonication method.

The particles exhibited semispherical shapes with sizes of approximately $350 \mathrm{~nm}$ depending on the content of $\alpha$-tocopherol and relatively narrow particle size distribu- tion, exhibiting acceptable stability. Successive conjugation and reconstitution process of $\alpha$-tocopherol and chitosan nanoparticles when including appropriate amount of $\alpha$ tocopherol was also observed. According to results, this procedure could be a facile method to prepare chitosan and $\alpha$-tocopherol nanoparticles.

\section{Acknowledgment}

The helpful advice of Dr. Reza Faridi-Majidi (Tehran University of Medical Sciences) is acknowledged.

\section{References}

[1] V. R. Sinha, A. K. Singla, S. Wadhawan et al., "Chitosan microspheres as a potential carrier for drugs," International Journal of Pharmaceutics, vol. 274, no. 1-2, pp. 1-33, 2004.

[2] R. D. Bhumkar and V. B. Pokharkar, "Studies on effect of $\mathrm{pH}$ on cross-linking of chitosan with sodium tripolyphosphate: a technical note," AAPS PharmSciTech, vol. 7, no. 2, article 50, 2006.

[3] G. Borchard, H. L. Lueßen, A. G. De Boer, J. C. Verhoef, C.M. Lehr, and H. E. Junginger, "The potential of mucoadhesive polymers in enhancing intestinal peptide drug absorption. III: effects of chitosan-glutamate and carbomer on epithelial tight junctions in vitro," Journal of Controlled Release, vol. 39, no. 2-3, pp. 131-138, 1996.

[4] S. Mansouri, P. Lavigne, K. Corsi, M. Benderdour, E. Beaumont, and J. C. Fernandes, "Chitosan-DNA nanoparticles as non-viral vectors in gene therapy: strategies to improve transfection efficacy," European Journal of Pharmaceutics and Biopharmaceutics, vol. 57, no. 1, pp. 1-8, 2004.

[5] D.-G. Kim, C. Choi, Y.-I. Jeong et al., "All-trans retinoic acid-associated low molecular weight water-soluble chitosan nanoparticles based on ion complex," Macromolecular Research, vol. 14, no. 1, pp. 66-72, 2006.

[6] M. Lee, J.-W. Nah, Y. Kwon, J. J. Koh, K. S. Ko, and S. W. Kim, "Water-soluble and low molecular weight chitosan-based plasmid DNA delivery," Pharmaceutical Research, vol. 18, no. 4, pp. 427-431, 2001.

[7] E. Reiter, Q. Jiang, and S. Christen, "Anti-inflammatory properties of $\alpha$ - and $\gamma$-tocopherol," Molecular Aspects of Medicine, vol. 28, no. 5-6, pp. 668-691, 2007.

[8] J. K. Lodge, "Vitamin E bioavailability in humans," Journal of Plant Physiology, vol. 162, no. 7, pp. 790-796, 2005.

[9] A. Dokoumetzidis and P. Macheras, "A century of dissolution research: from noyes and whitney to the biopharmaceutics classification system," International Journal of Pharmaceutics, vol. 321, no. 1-2, pp. 1-11, 2006.

[10] T. Kotyla, F. Kuo, V. Moolchandani, T. Wilson, and R. Nicolosi, "Increased bioavailability of a transdermal application of a nano-sized emulsion preparation," International Journal of Pharmaceutics, vol. 347, no. 1-2, pp. 144-148, 2008.

[11] P. B. Nielsen, A. Müllertz, T. Norling, and H. G. Kristensen, "The effect of $\alpha$-tocopherol on the in vitro solubilisation of lipophilic drugs," International Journal of Pharmaceutics, vol. 222, no. 2, pp. 217-224, 2001.

[12] A. Amani, P. York, H. Chrystyn, B. J. Clark, and D. Q. Do, "Determination of factors controlling the particle size in nanoemulsions using Artificial Neural Networks," European Journal of Pharmaceutical Sciences, vol. 35, no. 1-2, pp. 42-51, 2008. 
[13] M. L. Hans and A. M. Lowman, "Biodegradable nanoparticles for drug delivery and targeting," Current Opinion in Solid State and Materials Science, vol. 6, no. 4, pp. 319-327, 2002.

[14] W. Klaypradit and Y.-W. Huang, "Fish oil encapsulation with chitosan using ultrasonic atomizer," LWT-Food Science and Technology, vol. 41, no. 6, pp. 1133-1139, 2008.

[15] G. Ma, D. Yang, J. F. Kennedy, and J. Nie, "Synthesize and characterization of organic-soluble acylated chitosan," Carbohydrate Polymers, vol. 75, no. 3, pp. 390-394, 2009.

[16] Y. B. Che Man, W. Ammawath, and M. E. S. Mirghani, "Determining $\alpha$-tocopherol in refined bleached and deodorized palm olein by Fourier transform infrared spectroscopy," Food Chemistry, vol. 90, no. 1-2, pp. 323-327, 2005.

[17] A. Lienau, T. Glaser, M. Krucker et al., "Qualitative and quantitative analysis of tocopherols in toothpastes and gingival tissue employing HPLC NMR and HPLC MS coupling," Analytical Chemistry, vol. 74, no. 20, pp. 5192-5198, 2002. 

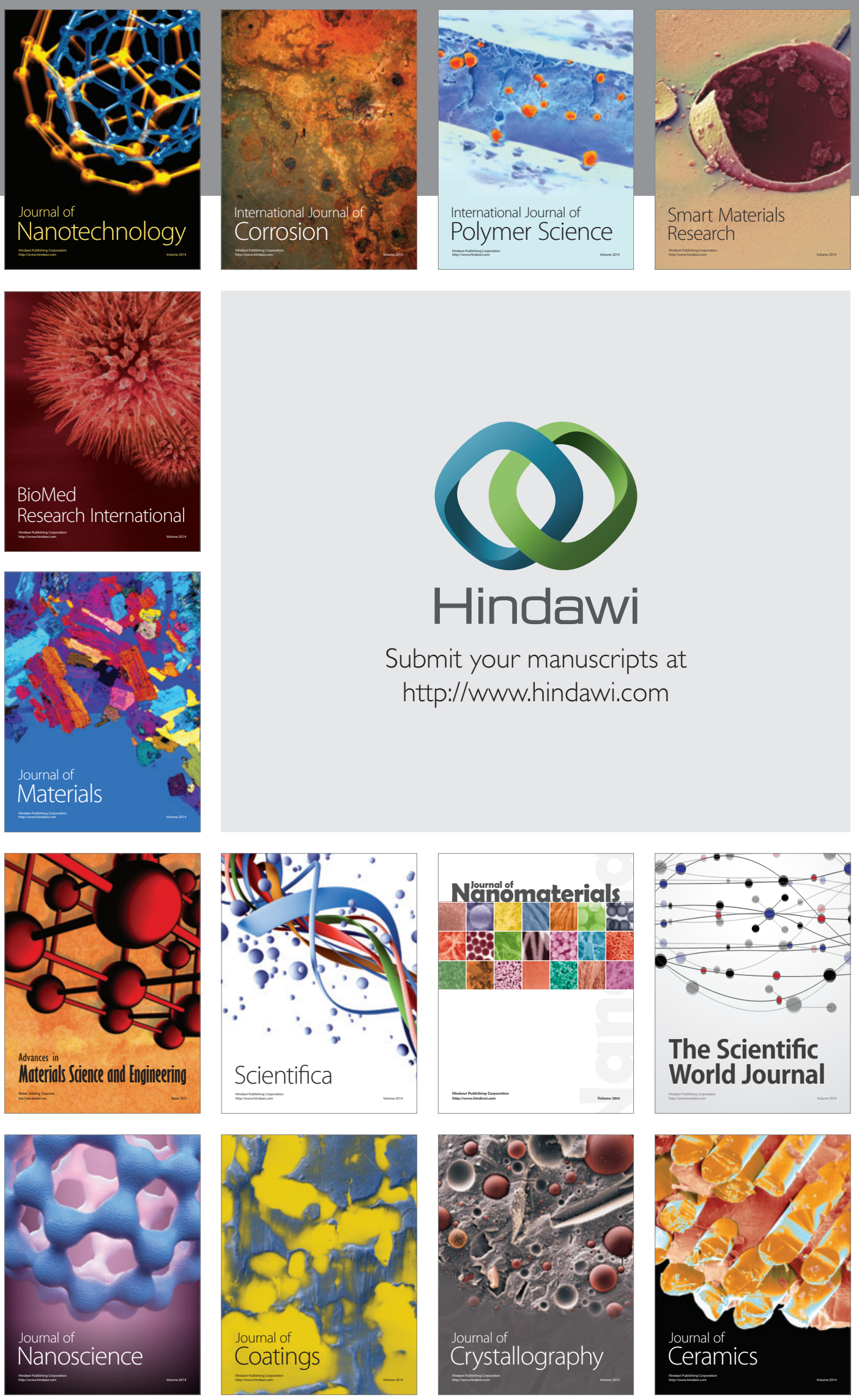

The Scientific World Journal

Submit your manuscripts at

http://www.hindawi.com

\section{World Journal}

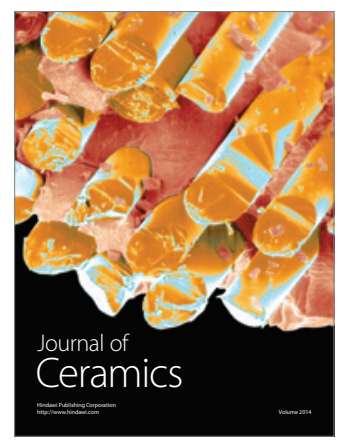

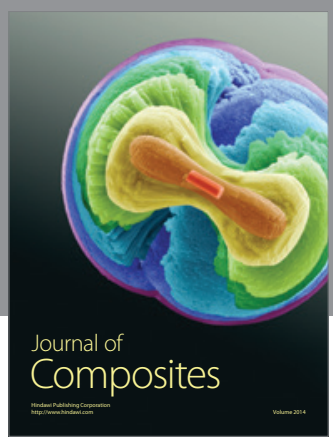
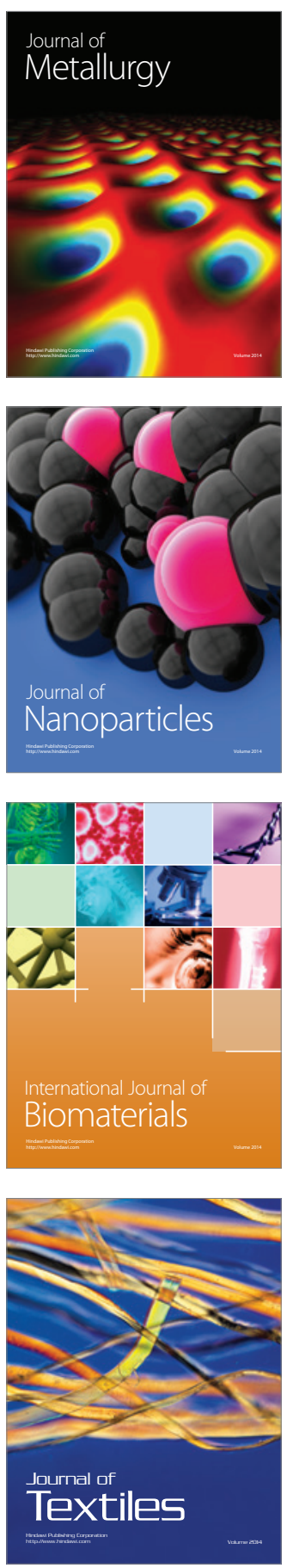